\title{
One-pot three-component synthesis of some new azo-pyrazoline derivatives
}

\author{
Farouq Emam Hawaiz *, Awaz Jamil Hussein and Mohammed Kareem Samad \\ Department of Chemistry, College of Education, University of Salahaddin-Hawler, Erbil 44001, Kurdistan Region, Iraq
}

*Corresponding author at: Department of Chemistry, College of Education, University of Salahaddin-Hawler, Erbil 44001, Kurdistan Region, Iraq. Tel.: +964.750.4605629. Fax: +964.750.4605629. E-mail address: farouqemam@yahoo.com (F.E. Hawaiz).

\section{ARTICLE INFORMATION}

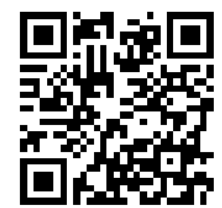

DOI: 10.5155 /eurjchem.5.2.233-236.979

Received: 30 November 2013

Received in revised form: 05 January 2014

Accepted: 06 January 2014

Online: 30 June 2014

\section{KEYWORDS}

\section{Cyclization}

Benzylation

Diazotization

Azo-pyrazoline

One-pot synthesis

Azo-acetophenone

\section{Introduction}

A one-pot synthesis, in which three [1-3], four [4] or more [5] components react in just one reactor to a final product is a synthetically useful method for improving the efficiency of chemical reaction, where interacting molecules subject to successive chemical reactions in just a single vessel. This is very useful for organic chemists, because of significantly shortens the reaction process by reducing the number of steps and avoiding a long separation processes and purification of chemical intermediates, would pay a way to green chemistry approach by save time and solvents for the purification processes, and increasing the proportion of output products [6].

A traditional synthesis of pyrazoline derivatives involve a two-step process, the Claisen-Schmidt condensation reaction of substituted acetophenones and substituted benzaldehydes to give chalcones [7], which undergo a subsequent cyclization reaction with hydrazines producing 2-pyrazolines [8]. Afterwards a number of synthetic approaches to synthesis of pyrazolines were reported, which include one-pot threecomponent condensation reaction $[9,10]$. Considerable attention has been focused on the synthesis of pyrazoline derivatives due to their wide spectrum of biological activity which include: anti-malarial [11], anti-fungal [12], antiinflammatory [13], analgesic [14], antileishmanial [15], antioxidant [16] and other antimicrobial activities [17,18]. Herein we have described the synthesis and characterization of some new azo-pyrazoline derivatives containing benzyloxy moiety through a one-pot three-component condensation reaction of azo-acetophenone, substituted benzaldehydes and phenylhydrazine.

\section{Experimental}

\subsection{Instrumentation}

Melting points were determined using an Electro thermal melting point apparatus, IR spectra were recorded on Shimadzu -FTIR Affinity-1, using $\mathrm{KBr}$ disc. ${ }^{1} \mathrm{H}$ NMR, ${ }^{13} \mathrm{C}$ NMR and ${ }^{13} \mathrm{C}$ DEPT 135 spectra were recorded on a Bruker Ultra Shield ( $300 \mathrm{MHz}$ ) with TMS and $\mathrm{CDCl}_{3}$ as an internal reference and solvent, respectively.

\subsection{Synthesis of 1-[4-\{(4-(4-chlorobenzyloxy)-2-methyl phenyl)diazenyl\} phenyl] ethanone (2)}

According to the modified procedure [19] a mixture of in situ prepared compound 1 [20] (9.14 g, $0.036 \mathrm{~mol}$ ), 4-chloro benzyl chloride ( $6.44 \mathrm{~g}, 0.04 \mathrm{~mol})$ and anhydrous $\mathrm{K}_{2} \mathrm{CO}_{3}(7.45 \mathrm{~g}$, $0.054 \mathrm{~mol}$ ), in ( $30 \mathrm{~mL}$ ) ethanol $96 \%$ was refluxed with stirring for $6 \mathrm{hrs}$. The cooled solution poured into water, the solid material immediately was obtained, then filtered off, washed several times with cold water dried and recrystallized from a mixture of xylene: ethanol $(1: 10, v: v)$ to give orange crystals of compound 2 (Scheme 1). 

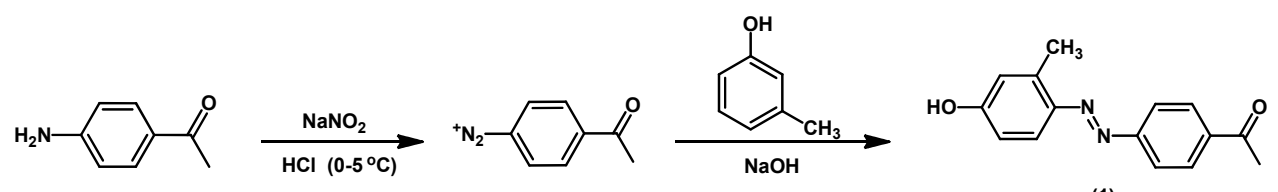

(1)
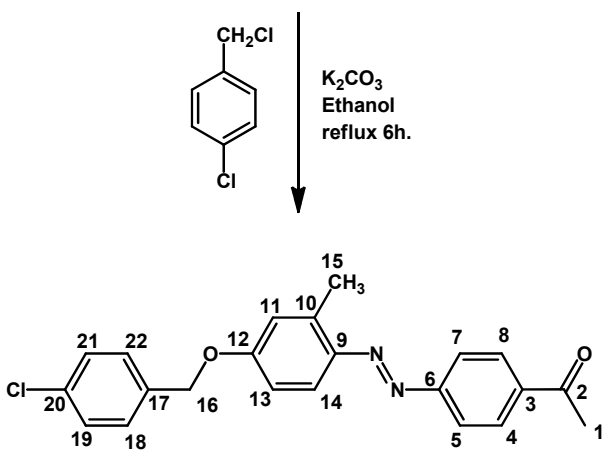

(2)
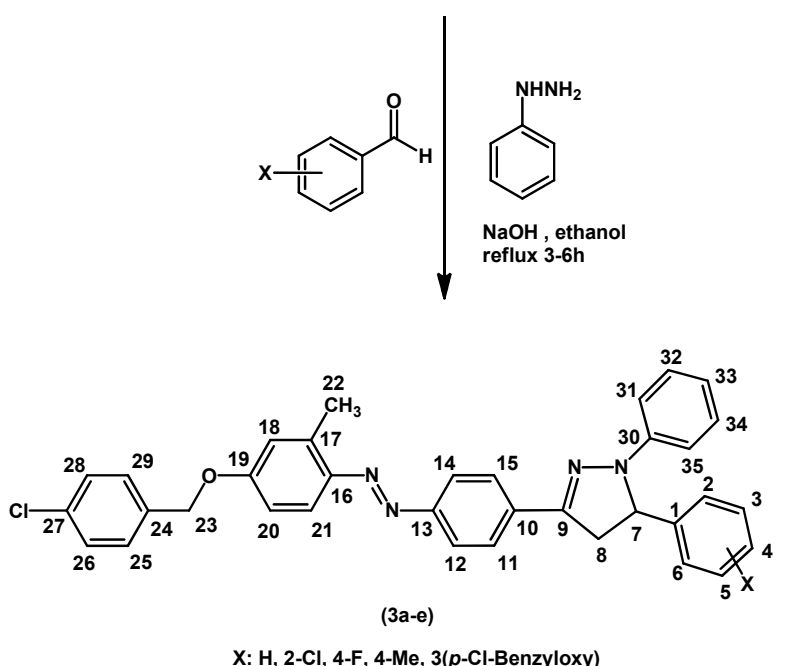

Scheme 1

Yield: 13.20 g, 97\%. M.p.: 139-141 ${ }^{\circ} \mathrm{C}$. IR $\left(\mathrm{KBr}, v, \mathrm{~cm}^{-1}\right): 1683$ $(\mathrm{C}=0), 1597$ (C=C). ${ }^{1} \mathrm{H}$ NMR (300 MHz, $\left.\mathrm{CDCl}_{3} \delta, \mathrm{ppm}\right): 2.30$ (s, $\left.3 \mathrm{H}, \mathrm{Ar}-\mathrm{CH}_{3}\right), 2.65\left(\mathrm{~s}, 3 \mathrm{H}, \mathrm{COCH}_{3}\right), 5.14\left(\mathrm{~s}, 2 \mathrm{H}, \mathrm{OCH}_{2}\right), 6.85-8.10$ (m, 11H, Ar-H'). ${ }^{13} \mathrm{C}$ NMR (75 MHz, $\left.\mathrm{CDCl}_{3} \delta, \mathrm{ppm}\right): 17.88\left(\mathrm{C}_{15}\right)$, $26.55\left(\mathrm{C}_{1}\right), 69.36\left(\mathrm{C}_{16}\right), 113.28\left(\mathrm{C}_{13}\right), 116.41\left(\mathrm{C}_{11}\right), 117.21\left(\mathrm{C}_{5,7}\right)$, $122.72\left(\mathrm{C}_{14}\right), 128.79\left(\mathrm{C}_{18,22}\right), 128.87\left(\mathrm{C}_{19,21}\right), 129.36\left(\mathrm{C}_{4,8}\right)$, $134.01\left(\mathrm{C}_{10}\right), 134.94\left(\mathrm{C}_{20}\right), 137.75\left(\mathrm{C}_{3}\right), 141.88\left(\mathrm{C}_{17}\right), 145.39$ (C9), $155.59\left(\mathrm{C}_{2}\right) .{ }^{13} \mathrm{C}$ DEPT 135 (75 MHz, $\left.\mathrm{CDCl}_{3} \delta, \mathrm{ppm}\right): 17.88$ ( $\left.\mathrm{C}_{15}\right), 26.55\left(\mathrm{C}_{1}\right), 69.36\left(\mathrm{C}_{16}\right), 113.28\left(\mathrm{C}_{13}\right), 116.41\left(\mathrm{C}_{11}\right), 117.21$ $\left(\mathrm{C}_{5}, 7\right), 122.72\left(\mathrm{C}_{14}\right), 128.79\left(\mathrm{C}_{18,22}\right), 128.87\left(\mathrm{C}_{19,21}\right), 129.36\left(\mathrm{C}_{4}\right.$, 8).

\subsection{One pot synthesis of pyrazolines, 3-\{4-[4-(4-chloro- benzyloxy)-2-methyl-phenylazo]-phenyl)-5-(substituted phenyl)-1-phenyl-2-pyrazolines (3a-e)}

A mixture of compound $\mathbf{2}(1 \mathrm{mmol})$, substituted benzaldehyde $(1 \mathrm{mmol}), \mathrm{NaOH}(4 \%, 3 \mathrm{mmol})$ and phenyl hydrazine $(1.5 \mathrm{mmol})$ was mixed together in $20 \mathrm{~mL}$ ethanol $96 \%$ and refluxed with stirring for appropriate time until completion the reaction which was monitored by TLC and colour change. The solution was cooled and the solid product was separated by suction filtration, washed several times with cold water dried and recrystallized from acetone to give pyrazolines (3a-e) (Scheme 1).

3-\{4-[4-(4-Chloro-benzyloxy)-2-methyl-phenylazo]-phenyl\}5-phenyl-1-phenyl-2-pyrazoline (3a): Yield: 78\%. M.p.: 181-183 ${ }^{\circ} \mathrm{C}$. Time: $4 \mathrm{~h}$. IR (KBr, v, cm-1): 1595(C=N), 1573, 1554 (C=C). ${ }^{1} \mathrm{H}$ NMR $\left(300 \mathrm{MHz}, \mathrm{CDCl}_{3} \delta, \mathrm{ppm}\right): 2.73$ (s, 3H, $-\mathrm{Ar}^{-} \mathrm{CH}_{3}-\mathrm{C}_{22}$ ), 3.22 (dd, $1 \mathrm{H}, \mathrm{CH}_{2}-\mathrm{H}_{\mathrm{a}}-\mathrm{C}_{8}$ ), 3.88 (dd, $1 \mathrm{H}, \mathrm{CH}_{2}-\mathrm{H}_{\mathrm{b}}-\mathrm{C}_{8}$ ), 5.08 (s, $2 \mathrm{H}-$ $\left.\mathrm{O}-\mathrm{CH}_{2}-\mathrm{C}_{23}\right), 5.33\left(\mathrm{dd}, 1 \mathrm{H}, \mathrm{CH}-\mathrm{H}_{\mathrm{x}}-\mathrm{C}_{7}\right), 6.78-7.85(\mathrm{~m}, 21 \mathrm{H}, \mathrm{Ar}-\mathrm{H})$.

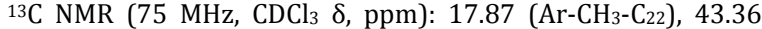
$\left(\mathrm{CH}_{2}-\mathrm{C}_{8}\right), 64.59\left(\mathrm{CH}-\mathrm{C}_{7}\right), 69.28\left(\mathrm{O}-\mathrm{CH}_{2} \mathrm{C}_{23}\right), 113.12\left(\mathrm{C}_{20}\right), 113.50$ ( $\left.\mathrm{C}_{18}\right), 116.36$ ( $\left.\mathrm{C}_{31,35}\right), 117.04$ ( $\left.\mathrm{C}_{33}\right), 119.42$ ( $\left.\mathrm{C}_{12,14}\right), 123.05\left(\mathrm{C}_{21}\right)$, $125.51\left(\mathrm{C}_{4}\right), 125.83\left(\mathrm{C}_{2,6}\right), 127.66\left(\mathrm{C}_{3,5}\right), 128.33\left(\mathrm{C}_{25,29}\right), 128.78$ ( $\left.\mathrm{C}_{26,28}\right), 128.81\left(\mathrm{C}_{11,15}\right), 128.94\left(\mathrm{C}_{32,34}\right), 129.19\left(\mathrm{C}_{17}\right), 133.91$ $\left(\mathrm{C}_{10}\right), 134.44\left(\mathrm{C}_{27}\right), 135.07\left(\mathrm{C}_{24}\right), 141.03\left(\mathrm{C}_{1}\right), 142.35\left(\mathrm{C}_{30}\right)$, 144.39 ( $\left.\mathrm{C}_{16}\right), 145.88$ (C9), $152.74\left(\mathrm{C}_{13}\right), 160.87\left(\mathrm{C}_{19}\right) .{ }^{13} \mathrm{C}$ DEPT $135\left(75 \mathrm{MHz}, \mathrm{CDCl}_{3} \delta, \mathrm{ppm}\right): 17.87\left(\mathrm{Ar}^{\left.-\mathrm{CH}_{3}-\mathrm{C}_{22}\right),-43.36\left(\mathrm{CH}_{2}-\right.}\right.$ $\left.\mathrm{C}_{8}\right), 64.59\left(\mathrm{CH}-\mathrm{C}_{7}\right),-69.28\left(0-\mathrm{CH}_{2} \mathrm{C}_{23}\right), 113.12\left(\mathrm{C}_{20}\right), 113.50$ $\left(\mathrm{C}_{18}\right), 116.36\left(\mathrm{C}_{31,35}\right), 117.04\left(\mathrm{C}_{33}\right), 119.42\left(\mathrm{C}_{12,14}\right), 123.05\left(\mathrm{C}_{21}\right)$, $125.51\left(\mathrm{C}_{4}\right), 125.83\left(\mathrm{C}_{2,6}\right), 127.66\left(\mathrm{C}_{3,5}\right), 128.33\left(\mathrm{C}_{25,29}\right), 128.78$ $\left(\mathrm{C}_{26,28}\right), 128.81\left(\mathrm{C}_{11,15}\right), 128.94\left(\mathrm{C}_{32,34}\right)$.

3-\{4-[4-(4-Chloro-benzyloxy)-2-methyl-phenylazo]-phenyl\}5-(o-chlorophenyl)-1-phenyl-2-pyrazoline (3b): Yield: 95\%. M.p.: 169-170 ${ }^{\circ}$ C. Time: 5 h. IR (KBr, v, cm-1): 1597 (C=N), 1575, 
$1546(\mathrm{C}=\mathrm{C}) .{ }^{1} \mathrm{H}$ NMR $\left(300 \mathrm{MHz}, \mathrm{CDCl}_{3} \delta, \mathrm{ppm}\right): 2.96$ (s, 3H, Ar$\mathrm{CH}_{3}-\mathrm{C}_{22}$ ), 3.20 (dd, $1 \mathrm{H}, \mathrm{CH}_{2}-\mathrm{H}_{\mathrm{a}}-\mathrm{C}_{8}$ ), 4.04 (dd, $1 \mathrm{H}, \mathrm{CH}_{2}-\mathrm{H}_{\mathrm{b}}-\mathrm{C}_{8}$ ), $5.11\left(\mathrm{~s}, 2 \mathrm{H}-\mathrm{O}-\mathrm{CH}_{2}-\mathrm{C}_{23}\right), 5.72\left(\mathrm{dd}, 1 \mathrm{H} \mathrm{CH}-\mathrm{H}_{\mathrm{x}}-\mathrm{C}_{7}\right), 6.67-7.80(\mathrm{~m}$, 20H, Ar-H). ${ }^{13} \mathrm{C}$ NMR $\left(75 \mathrm{MHz}, \mathrm{CDCl}_{3} \delta, \mathrm{ppm}\right): 17.86\left(\mathrm{Ar}^{-\mathrm{CH}_{3}-}\right.$ $\left.\mathrm{C}_{22}\right), 41.78\left(\mathrm{CH}_{2}-\mathrm{C}_{8}\right), 61.40\left(\mathrm{CH}-\mathrm{C}_{7}\right), 69.33\left(\mathrm{O}-\mathrm{CH}_{2} \mathrm{C}_{23}\right), 113.15$ $\left(\mathrm{C}_{20}\right), 113.29\left(\mathrm{C}_{18}\right), 116.40\left(\mathrm{C}_{31,35}\right), 117.08\left(\mathrm{C}_{33}\right), 119.57\left(\mathrm{C}_{12,14}\right)$, $123.06\left(\mathrm{C}_{21}\right), 126.35\left(\mathrm{C}_{5}\right), 127.30\left(\mathrm{C}_{4}\right), 127.67\left(\mathrm{C}_{6}\right), 128.21$ $\left(\mathrm{C}_{25,29}\right), 128.79\left(\mathrm{C}_{3}\right), 128.83\left(\mathrm{C}_{26,28}\right), 129.08\left(\mathrm{C}_{11,15}\right), 129.55$ $\left(\mathrm{C}_{32,34}\right), 129.95\left(\mathrm{C}_{17}\right), 132.88\left(\mathrm{C}_{2}\right), 133.02\left(\mathrm{C}_{27}\right), 134.30\left(\mathrm{C}_{10}\right)$, $136.82\left(\mathrm{C}_{24}\right), 139.01\left(\mathrm{C}_{1}\right), 144.21\left(\mathrm{C}_{30}\right), 145.92\left(\mathrm{C}_{16}\right), 146.85$ $\left(\mathrm{C}_{9}\right), 152.60\left(\mathrm{C}_{13}\right), 159.45\left(\mathrm{C}_{19}\right) .{ }^{13} \mathrm{C}$ DEPT $135\left(75 \mathrm{MHz}, \mathrm{CDCl}_{3} \delta\right.$, ppm): $17.86\left(\mathrm{Ar}_{-} \mathrm{CH}_{3}-\mathrm{C}_{22}\right),-41.78\left(\mathrm{CH}_{2}-\mathrm{C} 8\right), 61.40\left(\mathrm{CH}-\mathrm{C}_{7}\right),-$ $69.33\left(\mathrm{O}-\mathrm{CH}_{2} \mathrm{C}_{23}\right), 113.15\left(\mathrm{C}_{20}\right), 113.29\left(\mathrm{C}_{18}\right), 116.40\left(\mathrm{C}_{31,35}\right)$, $117.08\left(\mathrm{C}_{33}\right), 119.57\left(\mathrm{C}_{12,14}\right), 123.06\left(\mathrm{C}_{21}\right), 126.35\left(\mathrm{C}_{5}\right), 127.30$ $\left(\mathrm{C}_{4}\right), 127.67\left(\mathrm{C}_{6}\right), 128.21\left(\mathrm{C}_{25,29}\right), 128.79\left(\mathrm{C}_{3}\right), 128.83\left(\mathrm{C}_{26,28}\right)$, $129.08\left(\mathrm{C}_{11,15}\right), 129.55\left(\mathrm{C}_{32,34}\right)$.

3-\{4-[4-(4-Chloro-benzyloxy)-2-methyl-phenylazo]-phenyl\}5-(p-fluorophenyl)-1-phenyl-2-pyrazoline (3c): Yield: 77\%. M.p.: 181-183 ${ }^{\circ} \mathrm{C}$. Time: $5 \mathrm{~h}$. IR (KBr, v, cm$\left.{ }^{-1}\right): 1598(\mathrm{C}=\mathrm{N}), 1576$, $1546(\mathrm{C}=\mathrm{C}) .{ }^{1} \mathrm{H}$ NMR $\left(300 \mathrm{MHz}, \mathrm{CDCl}_{3} \delta, \mathrm{ppm}\right): 2.71$ (s, 3H, Ar$\mathrm{CH}_{3}-\mathrm{C}_{22}$ ), 3.12 (dd, $1 \mathrm{H}, \mathrm{CH}_{2}-\mathrm{H}_{\mathrm{a}}-\mathrm{C}_{8}$ ), 3.83 (dd, $1 \mathrm{H}, \mathrm{CH}_{2}-\mathrm{H}_{\mathrm{b}}-\mathrm{C}_{8}$ ), 5.28 (s, 2H, O- $\left.\mathrm{CH}_{2}-\mathrm{C}_{23}\right), 5.28$ (dd, $\left.1 \mathrm{H}, \mathrm{CH}-\mathrm{H}_{\mathrm{x}}-\mathrm{C}_{7}\right), 6.81-7.89$ (m, 20H, Ar-H). ${ }^{13} \mathrm{C}$ NMR $\left(75 \mathrm{MHz}, \mathrm{CDCl}_{3} \delta, \mathrm{ppm}\right): 17.90\left(\mathrm{Ar}^{-\mathrm{CH}_{3}-}\right.$ $\left.\mathrm{C}_{22}\right), 43.37\left(\mathrm{CH}_{2}-\mathrm{C}_{8}\right), 64.10\left(\mathrm{CH}-\mathrm{C}_{7}\right), 69.6\left(\mathrm{O}-\mathrm{CH}_{2}-\mathrm{C}_{23}\right), 113.16$ $\left(\mathrm{C}_{20}\right), 115.99\left(\mathrm{C}_{18}\right), 116.40\left(\mathrm{C}_{31,35}\right), 117.09\left(\mathrm{C}_{3,5}\right), 119.63\left(\mathrm{C}_{33}\right)$, $123.28\left(\mathrm{C}_{12,14}\right), 126.32\left(\mathrm{C}_{21}\right), 127.60\left(\mathrm{C}_{25,29}\right), 128.38\left(\mathrm{C}_{2,6}\right)$, $128.84\left(\mathrm{C}_{26,28}\right), 128.93\left(\mathrm{C}_{11,15}\right), 129.02\left(\mathrm{C}_{32,34}\right), 129.49\left(\mathrm{C}_{17}\right)$, $134.30\left(\mathrm{C}_{27}\right), 135.10\left(\mathrm{C}_{10}\right), 138.11\left(\mathrm{C}_{1}\right), 141.10\left(\mathrm{C}_{24}\right), 144.26$ $\left(\mathrm{C}_{30}\right), 145.47\left(\mathrm{C}_{16}\right), 145.92\left(\mathrm{C}_{9}\right), 152.84\left(\mathrm{C}_{13}\right), 160.14\left(\mathrm{C}_{4}\right)$, $160.82\left(\mathrm{C}_{19}\right) .{ }^{13} \mathrm{C}$ DEPT $135\left(75 \mathrm{MHz}, \mathrm{CDCl}_{3} \delta, \mathrm{ppm}\right): 17.90(\mathrm{Ar}-$ $\left.\mathrm{CH}_{3}-\mathrm{C}_{22}\right),-43.37\left(\mathrm{CH}_{2}-\mathrm{C}_{8}\right), 64.10\left(\mathrm{CH}-\mathrm{C}_{7}\right),-69.6\left(\mathrm{O}-\mathrm{CH}_{2}-\mathrm{C}_{23}\right)$, $113.16\left(\mathrm{C}_{20}\right), 115.99\left(\mathrm{C}_{18}\right), 116.40\left(\mathrm{C}_{31,35}\right), 117.09\left(\mathrm{C}_{3,5}\right), 119.63$ $\left(\mathrm{C}_{33}\right), 123.28\left(\mathrm{C}_{12,14}\right), 126.32\left(\mathrm{C}_{21}\right), 127.60\left(\mathrm{C}_{25,29}\right), 128.38\left(\mathrm{C}_{2,6}\right)$, $128.84\left(\mathrm{C}_{26,28}\right), 128.93\left(\mathrm{C}_{11,15}\right), 129.02\left(\mathrm{C}_{32,34}\right)$.

3-\{4-[4-(4-Chloro-benzyloxy)-2-methyl-phenylazo]-phenyl\}5-(p-methylphenyl)-1-phenyl-2-pyrazoline (3d): Yield: 83\%. M.p.: $119-121^{\circ} \mathrm{C}$. Time: $5 \mathrm{~h}$. IR (KBr, v, cm$\left.{ }^{-1}\right): 1596(\mathrm{C}=\mathrm{N}), 1572$, $1543(\mathrm{C}=\mathrm{C}) .{ }^{1} \mathrm{H}$ NMR $\left(300 \mathrm{MHz}, \mathrm{CDCl}_{3} \delta, \mathrm{ppm}\right): 2.65$ (s, 3H, Ar$\mathrm{CH}_{3}-\mathrm{C}_{22}$ ), 2.95 (s, 3H, $\mathrm{Ar}_{-} \mathrm{CH}_{3}-\mathrm{C}_{30}$ ), 3.17 (dd, $1 \mathrm{H}_{1} \mathrm{CH}_{2}-\mathrm{H}_{\mathrm{a}}-\mathrm{C}_{8}$ ), 3.85 (dd, $1 \mathrm{H}, \mathrm{CH}_{2}-\mathrm{H}_{\mathrm{b}}-\mathrm{C}_{8}$ ), 5.08 (s, $2 \mathrm{H}, \mathrm{O}-\mathrm{CH}_{2}-\mathrm{C}_{23}$ ), 5.30 (dd, $1 \mathrm{H}, \mathrm{CH}-$ $\left.\mathrm{H}_{\mathrm{x}}-\mathrm{C}_{7}\right), 6.77-8.10(\mathrm{~m}, 20 \mathrm{H}, \mathrm{Ar}-\mathrm{H}) .{ }^{13} \mathrm{C}$ NMR $\left(75 \mathrm{MHz}, \mathrm{CDCl}_{3} \delta\right.$,

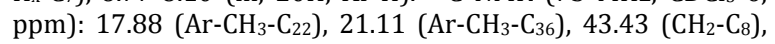
$64.42\left(\mathrm{CH}_{-} \mathrm{C}_{7}\right), 69.36\left(\mathrm{O}-\mathrm{CH}_{2} \mathrm{C}_{23}\right), 113.14\left(\mathrm{C}_{20}\right), 113.28\left(\mathrm{C}_{18}\right)$, 116.41 ( $\left.\mathrm{C}_{31,35}\right), 117.21\left(\mathrm{C}_{33}\right), 122.72\left(\mathrm{C}_{12,14}\right), 123.06\left(\mathrm{C}_{21}\right)$, $125.78\left(\mathrm{C}_{2,6}\right), 126.26\left(\mathrm{C}_{25,29}\right), 128.79\left(\mathrm{C}_{3,5}\right), 128.79\left(\mathrm{C}_{26,28}\right)$, $128.86\left(\mathrm{C}_{11,15}\right), 129.36\left(\mathrm{C}_{32,34}\right), 129.86\left(\mathrm{C}_{17}\right), 134.01\left(\mathrm{C}_{27}\right), 135.11$ $\left(\mathrm{C}_{10}\right), 137.75\left(\mathrm{C}_{4}\right), 139.42\left(\mathrm{C}_{24}\right), 141.88\left(\mathrm{C}_{1}\right), 144.46\left(\mathrm{C}_{30}\right)$, $145.40\left(\mathrm{C}_{16}\right), 145.89\left(\mathrm{C}_{9}\right), 152.74\left(\mathrm{C}_{13}\right), 161.53\left(\mathrm{C}_{19}\right) .{ }^{13} \mathrm{C}$ DEPT

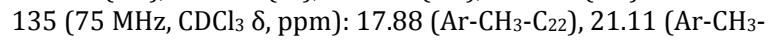
$\left.\mathrm{C}_{36}\right),-43.43\left(\mathrm{CH}_{2}-\mathrm{C}_{8}\right), 64.42\left(\mathrm{CH}-\mathrm{C}_{7}\right),-69.36\left(\mathrm{O}-\mathrm{CH}_{2} \mathrm{C}_{23}\right), 113.14$ $\left(\mathrm{C}_{20}\right), 113.28\left(\mathrm{C}_{18}\right), 116.41\left(\mathrm{C}_{31,35}\right), 117.21\left(\mathrm{C}_{33}\right), 122.72\left(\mathrm{C}_{12,14}\right)$, $123.06\left(\mathrm{C}_{21}\right), 125.78\left(\mathrm{C}_{2,6}\right), 126.26\left(\mathrm{C}_{25,29}\right), 128.79\left(\mathrm{C}_{3,5}\right), 128.79$ $\left(\mathrm{C}_{26,28}\right), 128.86\left(\mathrm{C}_{11,15}\right), 129.36\left(\mathrm{C}_{32,34}\right)$.

3-\{4-[4-(4-Chloro-benzyloxy)-2-methyl-phenylazo]-phenyl\}5-(m-(4-chlorobenzyloxy) phenyl)-1-phenyl-2-pyrazoline (3e): Yield: $86 \%$. M.p.: $164-166{ }^{\circ} \mathrm{C}$. Time: $3 \mathrm{~h}$. IR $\left(\mathrm{KBr}, \mathrm{v}, \mathrm{cm}^{-1}\right)$ : 1596 $(\mathrm{C}=\mathrm{N}), 1575,1542(\mathrm{C}=\mathrm{C}) .{ }^{1} \mathrm{H}$ NMR $\left(300 \mathrm{MHz}^{-\mathrm{CDCl}_{3} \delta} \mathrm{dpm}\right)$ : 2.73 (s, 3H, Ar- $\left.-\mathrm{CH}_{3}-\mathrm{C}_{22}\right), 3.19$ (dd, $\left.1 \mathrm{H}, \mathrm{CH}_{2}-\mathrm{H}_{\mathrm{a}}-\mathrm{C}_{8}\right), 3.85$ (dd, $1 \mathrm{H}$, $\left.\mathrm{CH}_{2}-\mathrm{H}_{\mathrm{b}}-\mathrm{C}_{8}\right), 4.96\left(\mathrm{~s}, 2 \mathrm{H}, \mathrm{O}-\mathrm{CH}_{2}-\mathrm{C}_{36}\right), 5.08\left(\mathrm{~s}, 2 \mathrm{H}, \mathrm{O}-\mathrm{CH}_{2}-\mathrm{C}_{23}\right), 5.51$ (dd, $\left.1 \mathrm{H}, \mathrm{CH}-\mathrm{H}_{\mathrm{x}}-\mathrm{C}_{7}\right), 6.80-7.87(\mathrm{~m}, 24 \mathrm{H}, \mathrm{Ar}-\mathrm{H}) .{ }^{13} \mathrm{C}$ NMR $(75 \mathrm{MHz}$,

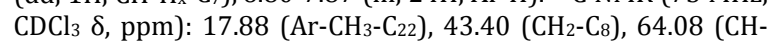
$\left.\mathrm{C}_{7}\right), 69.28\left(\mathrm{O}-\mathrm{CH}_{2}-\mathrm{C}_{36}\right), 69.32\left(\mathrm{O}-\mathrm{CH}_{2}-\mathrm{C}_{23}\right), 113.14\left(\mathrm{C}_{20}\right), 113.55$ $\left(\mathrm{C}_{18}\right), 115.45\left(\mathrm{C}_{31,35}\right), 116.39\left(\mathrm{C}_{3,5}\right), 117.06\left(\mathrm{C}_{33}\right), 119.42\left(\mathrm{C}_{12,14}\right)$, $123.07\left(\mathrm{C}_{21}\right), 126.27\left(\mathrm{C}_{2,6}\right), 127.13\left(\mathrm{C}_{25,29,38,42}\right), 128.74\left(\mathrm{C}_{26,28,39}\right.$, 41), 128.84 ( $\left.\mathrm{C}_{11,15}\right), 128.94\left(\mathrm{C}_{32,34}\right), 133.81\left(\mathrm{C}_{17}\right), 134.51\left(\mathrm{C}_{27,40}\right)$, $134.95\left(\mathrm{C}_{10}\right), 135.39\left(\mathrm{C}_{1}\right), 141.04\left(\mathrm{C}_{24,37}\right), 144.41\left(\mathrm{C}_{30}\right), 145.49$ $\left(\mathrm{C}_{16}\right), 145.90\left(\mathrm{C}_{9}\right), 152.76\left(\mathrm{C}_{13}\right), 158.03\left(\mathrm{C}_{4}\right), 160.90\left(\mathrm{C}_{19}\right) .{ }^{13} \mathrm{C}$

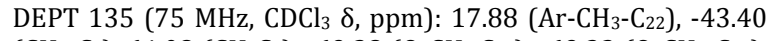
$\left(\mathrm{CH}_{2}-\mathrm{C}_{8}\right), 64.08\left(\mathrm{CH}-\mathrm{C}_{7}\right),-69.28\left(\mathrm{O}-\mathrm{CH}_{2}-\mathrm{C}_{36}\right),-69.32\left(\mathrm{O}-\mathrm{CH}_{2}-\mathrm{C}_{23}\right)$, $113.14\left(\mathrm{C}_{20}\right), 113.55\left(\mathrm{C}_{18}\right), 115.45\left(\mathrm{C}_{31,35}\right), 116.39\left(\mathrm{C}_{3,5}\right), 117.06$
$\left(\mathrm{C}_{33}\right), \quad 119.42 \quad\left(\mathrm{C}_{12,14}\right), \quad 123.07 \quad\left(\mathrm{C}_{21}\right), 126.27 \quad\left(\mathrm{C}_{2,6}\right), 127.13$ $\left(\mathrm{C}_{25,29,38,42}\right), 128.74\left(\mathrm{C}_{26,28,39,41}\right), 128.84\left(\mathrm{C}_{11,15}\right), 128.94\left(\mathrm{C}_{32,34}\right)$.

\section{Results and discussion}

In the last century, a huge number of pyrazoline and a little bit of azo-pyrazoline derivatives have been reported [21]. While there is no research in the literature for the synthesis of azo-pyrazoline derivatives through a one-pot three- component reaction process. Therefore, the aim of the work is to prepare some new azo-pyrazoline compounds in high yields and short reaction times avoiding separation and purification processes of intermediate chalcones by using a one-pot synthesis. In this study, firstly we have prepared the starting material azobenzyloxy-acetophenone (2), then used it as a main component with both phenylhydarzine and substituted benzaldehyde in a single vessel for one-pot synthesis approach of azo-pyrazolines (3a-e) as shown in Scheme 1. The newly synthesized compounds were characterized spectrally by using FT-IR, ${ }^{1} \mathrm{H}$ NMR, ${ }^{13} \mathrm{C}$ NMR and ${ }^{13} \mathrm{C}$ DEPT 135 spectra. In the IR spectrum of the starting material azo-benzyloxy (2), the disappearance of the hydroxyl group of compound $\mathbf{1}$, and shifting an absorption band of carbonyl group to $1683 \mathrm{~cm}^{-1}$, a strong band at 1597 $\mathrm{cm}^{-1}$ for $\mathrm{C}=\mathrm{C}$ of aromatic rings beside a two weak bands at 2924 and $2880 \mathrm{~cm}^{-1}$ attributed to the $\left(-\mathrm{CH}_{2}-\right)$ group of 4-benzyloxy moiety are confirmed the benzylation of hydroxyl group [22]. The ${ }^{1} \mathrm{H}$ NMR spectrum of compound (2) shows three singlet signals at $2.34,2.60$ and $5.20 \mathrm{ppm}$ belongs to the two $\left(-\mathrm{CH}_{3}\right)$ groups attached to the carbonyl and phenyl groups and two protons of $-0-\mathrm{CH}_{2}-$, respectively, a multiple signals at 6.8-8.2 ppm for 11 protons of three aromatic rings. The ${ }^{13} \mathrm{C}$ NMR spectrum showed eighteen singlets for eighteen types of carbons in different environments in the molecule. The ${ }^{13} \mathrm{C}$ DEPT 135 of azo-benzyloxy (2) showed nine upward signals for two $\left(\mathrm{CH}_{3}\right)$ groups and seven mono protonated carbons of aromatic rings and one downward signal corresponding to a diprotonated carbon atom of - $\mathrm{O}-\mathrm{CH}_{2}$ - group with disappearance of eight singlets for non-protonated carbon atoms [23].

In the IR spectra of the target molecule azo-pyrazoline the disappearance of carbonyl group band for both azoacetophenone and substituted benzaldehyde and appearance of two new bands around 1570 and $1550 \mathrm{~cm}^{-1}$ for imine system are good evidences for the formation of imine and the occurrence of cyclization reaction to give 2-pyrazoline. The ${ }^{1} \mathrm{H}$ NMR spectra of pyrazoline ring shows a very distinct signals for the protons attached to the $\mathrm{C}_{7}$ and $\mathrm{C}_{8}$ carbon atoms in the 2pyrazoline as an (ABX) spin system [24] which appeared three doublet to doublet (dd) signals around $\delta 3.2,3.8$, and $5.4 \mathrm{ppm}$ for two geminal and one vicinal protons prove a 2-pyrazoline structure. While the ${ }^{13} \mathrm{C}$ NMR spectrum shows two additional picks for two carbons $\left(\mathrm{C}_{7}\right.$ and $\mathrm{C}_{8}$ ) approximately at 45 and 65 $\mathrm{ppm}$ of the pyrazoline ring confirm the 2-pyrazoline structure [25]. Further support for the formation of the five member pyrazoline ring, is a downward signal of ${ }^{13} \mathrm{C}$ DEPT spectrum at $(-43 \mathrm{ppm})$ for the $\mathrm{CH}_{2}$ group of the ring in addition to the downward signal for $\mathrm{OCH}_{2}$ group at (-69 ppm).

\section{Conclusion}

The introduction of the azo-linkage, ortho to the acetyl group in acetophenone, will increases the conjugation and produce colored compounds that can be used as new starting materials for the preparation of different organic compounds. The preparation of azo-pyrazoline compounds from azoacetophenone, substituted benzaldehydes and phenylhydrazine can be achieved in high yields and short reaction times by using a one-pot synthesis method, via reducing the number of steps and avoiding a long separation processes and purification of chemical intermediates. 


\section{Acknowledgements}

This study was supported by Chemistry Department, College of Education, Salahaddin University-Hawler, Erbil, Kurdistan region, Iraq.

\section{References}

[1]. Hassan, V.; Ashraf, F. Molecules 2010, 15, 2972-2979.

[2]. Valentina, M.; Matthew, M. H.; Long, M.; Christine, M. C.; Andreas, P. T.; Dean, M. W. Synthesis 2002, 12, 1669-1674.

[3]. Santhosh, P.; Rajeswar, R. V. Org. Commun. 2012, 5(3), 143-149.

[4]. Mohammad, A.; Nasim, S.; Motahareh, H. Arkivoc 2012, 9, 13-20.

[5]. Hamad, M.; Khaled, D. K.; Aisha, Y. A.; Mohamed, H. E. Molecules 2010, 15, 6619-6629.

[6]. Javad, A.; Masoud, S.; Farhad, H.; Reza, M. Arkivoc 2006, 11, 47-58.

[7]. Mohammed, R. A.; Mohammed, H. R. K.; Vedula, G. S.; Nasreen, B.; Syed, A.; Yejella, R. P. Eur. J. Chem. 2012, 3(2), 186-190.

[8]. Bharat, K.; Vishal, P.; Sushma, R.; Ravi, K.; Tiwari, I. C. Int. J. Chem. Res. 2010, 2(1), 25-27.

[9]. Alam, M. M.; Marella, A.; Akhtar, M.; Husain, A.; Yar, M.; Shaquiquzzaman, M.; Tanwar, O. P.; Saha, R.; Khanna, S.; Shafi, S. Acta Pol. Pharm. 2013, 70(3), 435-441.

[10]. Lei-Lei, W.; Yi-Cen, G.; Ting, H.; Lei, Z.; Xing-Li, F.; Hai-Yan, F.; Hua, C.; Rui-Xiang, L. Synthesis 2012, 1577-1583.

[11]. Li, R.; Kenyon, G. L.; Cohen, F. E. J. Med. Chem. 1995, 38, 5031-5036.

[12]. Prasad, Y. R.; Kumar, P. P.; Kumar, P. R.; Rao, A. S. E-J. Chem. 2008, 5(1), 144-148.

[13]. Kouskoura, M.; Hadjipavlou, L. D.; Giakoumakou M. Med. Chem. 2008, 4, 586-596.

[14]. Sahu, S. K.; Banerjee, M.; Samantray, A.; Behera, A.; Azam, M. A. Trop. J. Pharm. Res. 2008, 7(2), 961-968.

[15]. Anees, A.; Siddiqui, M. D.; Azizur, R.; Shaharyar, M.; Ravinesh M. Chem. Sci. J. 2010, 8, 1-10.

[16]. Suthakaran, R.; Somasekhar, G.; Sredivi, C. H.; Marikannan, K. S.; Nagarajan, G. Asian J. Chem. 2007, 19(5), 3353-3362.

[17]. Ahmet, O.; Gulhan, T.; Zafer A. K. Turk. J. Chem. 2008, 32, 529-538.

[18]. Chovatla, Y. S.; Gandhi, S. P.; Gorde, P. L.; Bagade, S. B. Orient. J. Chem. 2010, 26(1), 275-278.

[19]. Ngaini, Z.; Haris, S. M.; Hussain, H.; Kamaruddin, K. World J. Chem. 2009, 4(1), 9-14.

[20]. Uhood, J.; Tarik, E. G.; Howraa, H. R. Molecules 2010, 15, 5620-5628

[21]. Veena, V. K.; Vijaya, K. D.; Venkata, R. C. Der Pharma Chem. 2012, 4(5), 2003-2008.

[22]. Farouq, E. H.; Mohammad, K. S. E-J. Chem. 2012, 9(3), 1613-1622.

[23]. Field, L. D.; Stern, S.; Kalman, J. R. Organic Structures from Spectra, $3^{\text {rd }}$ ed.; Wiley \& Sons: New York, 2005.

[24]. Assia, S.; Nouara Z.; Ouafa, D.; Mahieddine, M.; Kaddou, L. Eur. J. Chem. 2013, 4(3), 268-271.

[25]. Mamta, R.; Mohamad Y. Eur. J. Chem. 2012, 3(1), 21-25. 